\title{
Beitrag zur Lehre vom Glioma retinae.
}

\section{Von}

Dr. Th. Treitel, Privatdocent in Königsberg i. Pr.

Während die Lehre vom Glioma retinae klinisch in Wesentlichen abgeschlossen ist, sind in Bezug auf die pathologische Anatomie des Netzhautglioms noch verschiedene Detailfragen zu erledigen, z. B. in Bezug auf die Form der Gliomzellen und die Wege der heteroplastischen Erkrankung der übrigen Theile des Auges. Wenn schon aus diesem Grunde die Mittheilung eines einzelnen mit besonderer Berücksichtigung der fraglichen Punkte untersuchten Falles von Glioma retinae gerechtfertigt erscheint, so dürfte dies von dem vorliegenden anch deshalb gelten, weil er eine bisher garnicht oder nur ganz ausnahmsweise*)

*) In nenester Zeit hat Hänsell eine Mittheilung über gliomatöse Erkrankung des Glaskörpers gemacht; ich habe dieselbe nicht berücksichtigen können, da mir das Original (Bull. des quinze-vingts 1884) nicht zugänglich war. Ein kurzes Referat findet sich im Jahresbericht des Arohivs für Augenheilkunde, XV. Band, pag. 478; es geht aus demselben nicht ganz klar hervor, ob es sich in den von Hänsell untersuchten Bulbis um eine primäre oder metastatische Erkrankung des Corpus vitreum handelt. - 
beobachtete metastatische Affection des Glaskörpers zeigt. Diese konnte am Lebenden diagnosticirt und durch die pathologisch-anatomische Untersuchung bestätigt werden.

Die Beobachtung betrifft einen $2^{3 / 4}$ Jahre alten Knaben, der am 9. August 1885 zu mir gebracht wurde; die Mutter, welche das Kind gut beobachtes zu haben scheint, theilte mir mit, dass ihr bald nach der Geburt die weissliche Farbo der Pupille des linken Auges aufgefallen sei; eine weitero Yränderung des Auges sei erst eingetreten, als das Kind 1\% Jahr alt war. Ein Arzt in Russland habe nach einiger Zeit einen Einstich in das Auge gemacht, angeblich, um den Eiter zu entfermen, und vor drei Monaten habe man in Warschau das linke Auge enucleirt; nach der Beschreibung war dabei das Auge stark vergrössert, und die Geschwulst perforirt. Bald nach der Enucleation fing die Orbita an, sich mit Gesehwulstmasse zu füllen; das Recidiv wuchert jetzt stark aus der Augenhöhle hervor, die mit dem Tumor fest verwachsenen, sehr gedehnten und verbreiterten Lider vor sich herdrängend. Das andere Auge soll nach Angabe der Mutter vollkommen gesund sein, und der Knabe mit demselben gut sehen können. Aeusserlich konnte ich an diesem Auge nichts Abnormes erkennen, sah mich aber namentlich deswegen

Nach Einsendung des Manuscriptes konnte ich durch gütige Vermittelung des Herm Prof. Leber in die Arbeit von Hänsell Einsicht nehmen und mich davon überzengen, dass es sich bei ulen von diesem Antor anatomiseh untersuchten Fällen um eine secundäre Erkxankung des Glaskörpers bei Gliom der Retina handelt. Hänsell theilt drei Beobachtnngen mit, von denen namentlich die letzte interessant ist. Bei dieser fand sich mitten im wenig veränderten Glaskörper ein isolirter, etwa eine Linse grosser Gliomtumor; die erste Beobachtung betrifft aber nicht eine gliomatöse Infiltration, sondern nur eine Verdichtung des Gisaskörpers, eine Unwandlung desselben in fibrilläres Gewebe.

Hänsell citirt die ron Heitzmann auf dem Heidelberger Congress des Jahres 1883 über Veränderungen des Glaskörpers bei intraoculären Geschwülsten gemachten Mittheilungen.

Ob Heitzmann thatsächlich gliomatöse Degeneration des Glaskörpergewebes beobachtet hat, scheint mir ans seinen Angaben nicht vollkommen sicher hervorzugehen. Dagegen beanspruchen 
veranlasst, eine ophthalmoskopische Untersuchung vorzunehmen, um festzustellen, ob neuritische Veränderungen der Papille bestehen, und auf diese Weise eventnell einen Anhaltepunkt für das Vorhandensein metastatischer intracranieller Tumorbildung zn erhalten. Das sonstige Verhalten sprach garnicht für eine derartige Annahme; das Kind sah geradezu blühend aus und erschien vollkommen gesund; vor dem linken Ohre war eine kleine Drüse zu fühlen. Die Unterleibsorgane boten nichts Krankhaftes dar; über Kopfschmerzen soll der Knabe nicht geklagt und keine Symptome gezeigt haben, die auf ein Gehirnleiden hätten bezogen werden können. Dass ein solches trotzdem bestehen kann, ist ja allgemein bekannt; und in einem vor mehreren Jahren beobachteten Fall von Orbitalrecidiv von Gliom hatte ich gesehen, welch' wichtigen Fingerzeig die Papillitis des anderen anscheinend gesunden, sehtïchtigen

unser ganzes Interesse mehrere von Rompe in seiner Dissertation (Beitrag zur Kenntniss des Glioma retinae, Göttingen 1884) beschriebene Gliomfälle, anf die Herr Prof. Leber mich anfmerksam zu machen die Freundlichkeit hatte. Unter den vier von Rompe publicirten Beobachtungen wurden in der ersten und vierten - ebenso wie in der von mir oben mitgetheilten - klinisch und pathologisch-anatomisch zahlreiche miliare Gliomknötchen im Glaskörper festgestellt. Auch hat Herr Prof. Leber nach brieflicher Mittheilung noch in einem anderen Präparat gliomatöse Infiltration der Zonula beobachtet. Demnach wird es wahrseheinlich, dass eine secundäre gliomatöse Erkrankung des Corpus vitrenm bei Netzhautgliom nicht so selten vorkommt, als es die bisherigen Darstellungen haben annehmen lassen. Ich möchte aber darauf schon hier besonders hinweisen, dass bei Sarcom der Chorioidea heteroplatische Degeneration des Glaskörpers meines Wissens bisher nicht beschrieben worden ist.

Es dürfte die Leser des Archivs, denen zum Theil die Rompe'sche Dissertation vielleicht ebenso wie mir entgangen ist, interessiren, dass daselbst über Impf- und Züchtungsversuche mit Gliompartikelchen berichtet wird, die von Herrn Prof. Leber mit Berïcksichtigung aller jetzt ïblichen Kautelen angestellt worden sind. Beide Versuchsreihen fielen negativ aus. Aus den an Kaninchenaugen angestellten Impfversuchen darf aber, wie der Verfasser mit Recht hervorhebt, nur geschlossen werden, dass das Netzhautgliom des Menschen auf das Kaninchenauge nicht übertragbar ist, keineswegs aher, dass diese Frage für den Menschen damit entschieden wird. 
Auges für die Diagnose der Ausbreitung des Glioms in dem intracraniellen Raum und damit für dje Prognose geben kann. Bei der Untersuchung des rechten Auges fanden sich nun andere interessante Veränderungen; wenn diese wegen der Unruhe des Kindes auch nicht sehr eingehend beobachtet werden konnten, so liess sich doch bei miederholten. Untersuchungen Folgendes feststellen. Erstens erschien die Papille und ihre nächste Umgebung durch eine gleichmässig, intensiv weisse gefässlose Masse verdeckt; die Begrenzung derselben ganz unregelmässig, die neben derselben wie abgeschnitten endigenden Retinalgefässe normal; ebenso der übrige Hintergrund. Zweitens reflectirte der vordere Abschnitt der inneren Bulbushälffe bei seitlicher Beleuchtung und bei ophthalmoskopischer Tntersuchung intensiv weisses Licht; die weisse anscheinend prominirende Masse liess keine Gefässe erkennen. Drittens zeigte sich der Glaskörper mit unzähligen, sehr kleinen, im Allgemeinen rundlichen Flocken resp. Knötchen durchsetzt, die weisses licht von demselben Farbenton und derselben Intensität reflectirten, wie die Masse vor der Papille und im vorderen inneren Abschnitt des Augapfels. Diese Gebilde fanden sich in der ganzen Breite des Glaskörpers and anscheinend in verschiedener Tiefe desselben; sie flottirten bei Bewegungen des Auges erheblich, behielten aber dabei ihre relative Lage zu einander; ich nahm daher an, dass sie an Membranen des Glaskörpers hafteten.

Die Pupille war zum Zweck der genaueren Untersuchung durch Atropin dilatirt worden, und man erkannte nun auch bei diffusem Tageslicht einen hellen Schein in der Tiefe des Auges. Dass das Kind mit dem rechten Auge noch gut sehen konnte, ging aus dem Verhalten desselben mit Sicherheit hervor; eine genane Prüfung der Sehschärfe war selbstverständlich ansgesehlossen.

Die Mutter des kleinen Patienten wünschte, dass das Recidiv entfernt würde, obwohl sie die Gefahr einer derartigen Operation kannte. $A m$ 13. August führte ich in tiefer Narkose die Esenteratio orbitae incl. Entfernung des Periost und der Augenlider aus; das Periost liess sich sehr leicht loslösen; die Wände der Augenhöhle erwiesen sich als ganz normal bis auf eine Stelle der unteren, dicht hinter der Eintrittsstelle des Nervus infraorbitalis; hier war der Knochen 
rauh und etwas eingedrückt. Die Operation war unter streng antiseptischen Cautelen ausgeführt worden (zum Bespülen des Operationsterrains und zum Abtupfen der Wunde wurde Sublimat $0,4 / 1000$ verwendet); der Verband wurde zum ersten Male am 16. gewechselt, und ich glaubte um so mehr auf einen weiteren günstigen Verlauf hoffen zu dürfen, da ich die Orbita ganz frei von Secret fand, und der Kranke munter war; aber am 17. Morgens traten ausgesprochene meningitische Symptome ein, das Kind wurde bald somnulent und starb am 18. August Nachmittag gegen 5 Uhr. Am 17. hatte ich noch eine ophthalmoskopische Untersuchung des rechten Auges angestellt; dabei erschienen mir die Retinalvenen ein wenig geschlängelt und verdickt, aber nicht mit dunklerem Blut gefüllt.

Ich füge hier das Protocoll der in dem hiesigen pathologischanatomischen Institut von Herrn Dr. Ortmann ausgeführten Section ein: Sehr gut genährte männliche Kindesleiche. Die linke leere Augenhöhle auffallend gross in allen Dimensionen. Die ihres Periostes beraubten Knochenränder zeigen sich überall bedeckt von einer $1 / 2-1 \mathrm{Mm}$. dicken Schicht weithen grauröthlichen Granulationsgewebes, das mit der Sonde leicht zu durchstossen und von der Knochenunterlage abhebbar ist. Die den Boden der Orbita bildende Platte erscheint sehr dünn und stellenweise eingedrückt. Der Sulcus infraorbitalis ist weiter als normal, der in ihm liegende Stumpf des $\mathbf{N}$. infraorbitalis dick, grauroth, anscheinend geschwulstartig entartet. In der Tiefe der Augenhöhle ist das Granulationsgewebe bedeckt mit einer dünnen Schicht dickflüssigen, schmutzig-bräunlichen Fiters; derselbe Eiter haftet an der Spitze des aus der Orbita entfernten Wattetampons. Nach Eröffnung der Schädelhöhle erscheint der Blutgehalt der Dura mater und der Diploe des Schädeldaches ein beträchtlicher, der Sinus longitudinalis angefüllt mit weichem Cruor und Speckhautgerinnseln; die weichen Hirnhäute succulent, getrübt, die Gefässe anch über den Stirnlappen beträchtlich angefüllt. Entlang der linken Fossa sylvii, längs den Gefässen zu beiden Seiten, die Pia mit gelbem Eiter infiltrirt, besonders stark nach der Basis zu. Bei dem Versuche, die Vorderhörner aus der vorderen Sehädelgrube herauszuheben, zeigt sich innerhalb des Duralraumes eine den linken Opticus rom Chiasma an einnehmende Geschwulst von grauröthlicher Farbe und sehr 
weicher Consistellz. Die Geschwulst ist etwa von der Grösso einer Haselnuss, etwas abgeplattet und liegt dem Keilbeinkörper auf. Nach der Abtrennung der Dura von ihren Verbindungen mit der Schädelbasis lässt sich der geschwulstartig entartete Stumpf des linken Opticus aus dem etwa kleinfingerdick verbreiterten Foramen opt. herausziehen. Der Nerv ist in seinem ganzen. Verlanfe vom Chiasma bis zur äusseren Oeffnung des For. opt. aufgegangen in dieselbe grauröthliche, sehr weiche, suceulente Geschwulstmasse. Am Chiasma grenzt sich die Neubildung scharf von der weissen Nervensubstanz ab. An der äusseren Fläche der Dura und auf dem Knochen in der Umgebung des For. opt. ist keine Spur von Eiter wahrzunehmen, ebenso wenig um oder innerhalb der Gesehwulst. Der rechte Opticus in seinem ganzen Verlaufe durchaus normal. An den Gefässen der Hirnbasis und den Sinus der Dura keine Veränderung. Die Hirnsubstanz sehr woich, suceulent, die Windungen etwas abgeplattet. In der mittleren und hinteren Schädelgrube bleibt nach Herausnahme des Hirns ziemlich reicher Liquor cerebrospinalis zurück; die Ventrikel etwas weiter als normal, ebenfalls reichlichen Liquor enthaltend. In den übrigen Organen findet sich nichts Bemerkenswerthes.

Der rechte Augapfel war $1 / 2$ Strnde p. mortem enucleirt und sofort untersucht worden: er hat normale Form und Grösse, sein sagittaler Durchmesser beträgt $24 \mathrm{Mm}$, der verticale $20 \mathrm{Mm}$.

Der Bulbus wird frisch eröffinet durch einen dicht neben der Cornea in der Sclera verlaufenden circulären Schmitt. Nach Abtragung der Hornhaut und dor daran haftenden Iris, wird die Linse, welche übrigens am Glaskörper ziemlich fest haftet, entleert; sie ist ganz klar und erleidet bei der Herausnahme keine Formveränderung. Dabei fliesst eine geringe Menge ganz klaren und normaler Weise consistenten Glaskörpers aus. Man sieht jetzt in der ganzen vorderen Fläche des Glaskörpers äusserst zablreiche, sehr kleine weisse Punkte resp. Kügelchen; sie haften an zarten, bei Bewegungen des Auges flottirenden Membranen. Im nasalen Abschnitt der vorderen Bulbushälfte präsentirt sich ein weisser, nicht vascularisirter Tumor, dessen hintere Grenze nicht genau festgestellt werden kann; seine Oberfäche ist uneben. Der Augapfel 
wird möglichst schnell nach der Eröffnung, nach höchstens 5 Minuten, in Müller'sche Flüssigkeit eingelegt, gleichzeitig mit der Hornhaut, Iris und Linse.

Einige von den weissen. Kügelchen des Glaskörpers werden frisch (in Glaskörpersubstanz) untersucht; sie zeigen sich zusammengesetzt aus granulirten rundlichen Zellen, zum Theil von der Grösse weisser Blutkörperchen, zum Theil von erheblicherem Umfang. Die Zwischensubstanz erscheint stellenweise ganz homogen, stellenweise granulirt. Nach Zusatz ron Eissigsäure treten Kerne hervor, die fast die ganze Zelle einnehmen. Blutgefässe sind in den untersuchten Stücken nicht zu sehen. Zwischen den Geschwulstzellen finden sich zahlreiche Fettkörnchenzellen. Die weisse Substanz ist sehr zäh, sie lässt sich in lange Fäden ausziehen. Nach achtwöchentlicher Härtung wird das Auge einer genauen Untersuchung unterworfen. Der Opticusstumpf wird in einer geringen Entfernung von der Sclera durchsehnitten, und darauf der Bulbus im horizontalen Meridian halbirt, die untere Hälfte aufbewahrt, die obere vollkommen entwässert und dann in Alkohol gehärtet. Es zeigt sich nun in der oberen. Hälfte des Bulbus der ganze nasale Abschnitt von einem soliden Tumor eingenommen; er bedeckt das Corpus ciliare und endigt ca. $3 \mathrm{Mm}$. vor der Papille; seine Farbe erscheint grauweiss, seine Oberffäche unregelmässig convex; der Tumor wird im hinteren Augapfelabschnitt allmählich ganz flach; eine ebenso beschaffene, ebenfalls sehr dünne Schicht des Tumors bedeckt, ähnlich einer Kugelschale, fast die ganze temporale Hälfte der Netzhaut, sie endigt hier $4 \mathrm{Mm}$. von der Ora serrata in einer unregelmässigen Linie. Diese Schicht scheint auf der Retina zu liegen ohne innigeren Zusammenhang mit derselben; sie lässt sich wenigstens stellenweise bei vorsichtiger Führung der Nadel von der Retina abheben; dabei zeigen sich in dieser einzelne sehr kleine, kaum prominirende, grauweisse Knötchen eingelagert. Die Geschwulstschicht der temporalen Netzhauthälfte hängt continuirlich zusammen einerseits mit der gleichen Schicht auf der nasalen Netzhauthälfte, andererseits mit einer circa $1 \mathrm{Mm}$. dicken Masse, welche die ganze Papille bedeckt; der Contour dieser Masse gegen die Papille erscheint makroskopisch ziemlich scharf, ihre Oberfläche gegen den Glaskörper ganz unregelmässig. In der Ge- 
schwulst sind nirgends Gefasse zu sehen. Von der Oberfläche des Geschwalsttnotens ziehon zahlreiche, mit einander zusammenbängende, durchscheinende, grauliche Glaskörpermembranen in der Richtung nach aussen und vorn zur Ora serrata und dem Corpus ciljare der temporalen Bulbushalfte, wo sie sich fest inseriren. Diese Nembranen sind mit unendlich vielen, äusserst kleinen, weisslichen Knötchen besetzt, die namentlich oben, in der Nähe der Netzhaut sehr dicht liegen. Die Selera und Chorioidea erscheinen normal; die Retina ist stellenweise von der Chorioidea etwas abgehoben, aber offenbar nur in Folge der Präparation. Der Quer und Längsschnitt des am Bulbus haftenden $\mathrm{N}$. opticus sehen vollkommen gesund. aus, die Papille ein wenig geschwellt, ebenso die angrenzende Retina. Vom oberen Rande dor auf der Papille liegenden Geschwulstschicht verlüuft ein kurzer, dünner, recht fester Faden nach dem hinteren Abschnitt des Tumors; er erscheint weisslich, nur neben der Papille bräunlich. (Der kleine Faden ging leider verloren und konnte daher nicht mikroskopisch untersucht werden.)

Bei der makroskopischen Beobachtung zeigt sich in der u a ter en Hälfte des Bulbus eine Fortsetzung des Tumors, von der Grösse einer lleinen Erbse; or liegt unmittelbar am Corpus eiliare; nur ein kleiner, angrenzender Abschnitt der Netzlant ist von einer dïnnen Sehicht der Geschwulst bedeckt. In der unteren Halfte des Bulbus finden sich noeh zahlreichere Glaskörpermembranen, als in der oberen, und zwar nieht allein im vorderen, sondern auch im hinteren Abschnitt; der mittlere Theil des Glaskörperraumes ist in dem erhärteter, eröffneten Ange vollkommen leer. Alle diese Membranen sind mit zahllosen Eleinen Geschwulstknötchen besetzt, von denen einzelne fast die Grösse eines kleinen Stecknadelkopfes erreichen. Im Uebrigen ist zu bemerken, dass der Querschnitt der Netzhaut etwas nach aussen rom hinteren $\mathrm{Pol}$ an drei Stellen in Folge kleiner disseminirter Geschwulstherde verdickt und gelblich erscheint. Die Oberfläche der Papille ist von einer sehr dümnen Schicht von Geschwulst bedeckt.

Der makroskopische Befund ergiebt also: einen Tumor im vorderen inneren oberen Quadranten, der sich in Form einer sehr dünnen Schicht fast über die ganze Netzhaut 
der oberen Hälfte des Augapfels und über die Papille bis zu ihrem unteren Rande ausbreitet, ferner zahlreiche disseminirte kleine Infiltrate der Netzhaut, geringe Anschwellung der Papille, und mit unzähligen sehr kleinen Geschwulstknötchen besetzte Glaskörpermembranen, besonders in den vorderen und hinteren Parthien des Glaskörpers.

Bei der mikroskopischen Untersuchung erwies sich der am Bulbus haftende Abschnitt des N. opticus bis zur Lamina cribrosa hin als vollkommen normal; ebenso die temporale Hälfte der Papille; ihre nasale Hälfte in geringer Ausdehnung von dem Gliom infiltrirt; die auf der Papille liegende Schicht der Geschwulst hängt mit der Oberfläche der nasalen Papillenhälfte innig zusammen, während sie die temporale Hälfte nur locker bedeckt und an den feinen Schnitten sich von derselben abgelöst hat. Die gliomatöse Infiltration erstreckt sich in der Richtung nach der Lamina cribosa, die sie nirgends erreicht, und in das Anfangsstück der Nerrenfaserschicht der Retina; sie bildet hier einen kleinen Knoten, der mit scharfem, convexen Contour bis dicht an die Ganglienschicht heranreicht, ohne in diese einzudringen. Die Grenzlinie gegen die tieferen Parthien der Papille erscheint ganz unregelmässig, indem einzelne zapfenförmige Fortsätze sich in die Nervenfaserbündel einsenken. Die Retina neben der Papille zeigt sich, abgesehen von der eben genannten Veränderung, ganz normal, die Stäbchenschicht sehr gut erhalten.

An Meridionalschnitten durch den vorderen Abschnitt des Bulbus in der Gegend des Tumors erscheint der Musculus und die Proc. ciliares etwas reicher an Kernen als in der Norm, sonst unverändert; neben dem glatten Theil des Corpus eiliare liegen zahlreiche rundliche Gliomberde von 0,02 bis $0,2 \mathrm{Mm}$. Durchmesser, zum Theil in rothen Blutkörperchen eingebettet. Einige von diesen Herden sind dadurch ausgezeichnet, dass sie genau im Centrum den Querschnitt eines kleinen Gefässes, dessen Wand deutlich ausgebildet ist, erkennen lassen; solche gefässhaltige Knötchen finden sich nur in der Nähe des Corpus ciliare und des Tumors, auf einzelnen Schnitten mehrere neben einander; sie sind wohl sicher als 
die vordersten Parthien des Haupttumors anzusehen und aur anf den feinen Meridionalschnitten scheinbar isolirt; hierfür spricht auch die Beobachtung, dass in den angrenzenden Theilen des Haupttumors Gefässe von derselben Beschaffenheit zu sehen sind.

Alle Glaskörperherde bestehen aus dicht gedrängten Gliomzellen und sind ganz gefässlos.

Die Pars ciliaris retinae und die an die Ora serrata angrenzende Retina erscheint normal, in einer Ausdehnung von ca. $3 \mathrm{Mm}$; dann schwillt die Netzhaut allmählich an in Folge von gliomatöser Infiltration der Nervenfaserschicht; dabei orscheinen anfangs dio übrigen Schichten der Retina ganz unverändert. Anf den Meridionalschnitten bildet der Geschwulstherd der Nervenfaserschicht meist ein Dreieck, dessen Spitze nach der Ora serrata gerichtet ist, nur ausnahmsweise einen rundlichen, nach vorn convexen Herd, Der bis dahin geradlinige Contour der Limitans externa erscheint nun schwach convex nach der Chorioidea, offenbar deshalb, weil dureh die verdickte Faserschicht die ganze übrige Netzhant gegen die Aderhaut verschoben ist. Die Degeneration ist anf die Nervenfaserschicht beschränkt in einer Ausdehnung von $0,07 \mathrm{Mm}$, und verbreitet sich dann schnell auf die ganze Dicke der Retina, deren Querschnitt bald sehr erheblich zunimmt und in denjenigen des Tumors ubergeht. Dieser ist ans dicht gedrängten, einen grossen Kern enthaltenden Zellen zusammengesetzt und von sehr weiten, äusserst dünnwandigen Gefässen durchzogen. Der Tumor dehnt sich nach vorn bis in die Ebene der Ansatzstelle des Musculus ciliaris aus, indem er sich über die normale Retina legt; dabei ist die Faserschicht derselben ist stellenweise eingedrüekt, usurirt; die Limitans interna scheint aber nicht durchbrochen $z u$ sein, wenigstens zeigt sich ihr Contour überall, wo sich der Gesehwulstquesrchnitt von der Retina in Folge der Präparation gelockert hat, ganz scharf.

Um noch einen weiteren Einblick in die Veränderungen der Retina an der Grenze der Geschwulst zu erhalten, wurden feine Schnitte aus den oberen Parthien der letzteren angefertigt, 
und zwar gleichzeitig aus Abschnitten der Netzhaut mit kleinen isolirten Knötchen. Diese Schnitte wurden parallel dem Aequator angelegt, um das Verhalten der Radiärfasern genauer beurtheilen zu können. Was die kleinen Netzhautknötchen anbetrifft, so zeigen sie mikroskopisch eine länglich ovale Gestalt und bestehen aus dicht aneinander gedrängten Gliomzellen; diese kleinen Herde liegen in der Nervenfaserschicht; die innere und äussere Körner-, die äussere granulirte and die Stäbchenschicht erscheinen ganz normal, sind auch nicht verbogen. Die innere granulirte Schicht ist schmäler und die Ganglienschieht ein wenig dicker als in der angrenzenden, gesunden Retina, die Faserschicht fast um das Dreifache verbreitert, hauptsächlich in der Richtung nach dem Glaskörper. Auf der nach dem Tumor gerichteten Seite des Knötchens erfolgt der Uebergang der normalen Retina in die infiltrirte fast ganz plötzlich, auf der entgegengesetzten zeigt sich als erste Veränderung eine Anschwellung und Verlängerung der Radiärfasern; diese nehmen je nüher dem Knötchen umso mehr zu; im Bereiche des letzteren scheinen die Müller'schen Fasern entsprechend der Verdickung der Nervenfaserschicht sehr erheblich verlängert zu sein.

An der Grenze der Geschwulst ist nur die Nervenfaserschicht infiltrirt bei durchaus normalem Verhalten der übrigen Schichten; nur an wenigen Stellen der Grenze des Tumors findet sich die Infiltration mit Gliomzellen glechicitig in der Nervenfaser- und in den Körnerschichten, besonders in der inneren Körnerschicht*).

Diese Schnitte wurden ausserdem mit besonderer Rücksicht auf das Verhalten der feinen Gefässe und der Capillaren untersucht; es zeigten sich keine bemerkenswerthen Ver-

*) Die anatomisschen Veränderungen in den Grenzparthien der Geschwulst scheinen den Schluss zu gestatten, dass die Nervenfaserschicht den zuerst erkrankten Abschnitt der Retina darstellt. Dieser Schluss ist aber nicht ganz einwandfrei. Die Infiltration an den Grenzen der Geschwulst braucht ebenso wenig wie ein isolirter metastatischer Netzhautherd, dieselbe Netzhautschicht 
änderungen derselben, insbesondere wurde oine Ansammlung von Geschwulstzellen in den perirasculären Lymphräumen nicht aufgefunden.

Zupfpräparate aus verschiedenen Theilen der Geschwulst lassen an der überwiegenden Mehrzahl der Zellen Fortsätze erkennen; diese Fortsătze sind meist kurz, dabei entweder zugespitzt oder plump, selten fadenförmig. Nur wenige Zellen sind mit relativ langen Fortsätzen versehen, von der zweibis dreifachen Länge der Zellen; nur eine einzige Zelle mit verzweigten Ausläufern wurde aufgefunden. Die meisten Zellen haben zwei Fortsätze, an gegenuber gelegenen Stellen, sie sehen daher bipolaren Ganglienzellen selrr ähnlich. Die Zellen sind so gross wie weisse Blutkörperchen, viele auch etwas umfangreicher, sie enthalten einen grossen Kern, der von einem schmalen Protoplasmaring umschlossen ist; das Protoplasma erscheint anffallend glänzend sowohl in der unmittelbaren Umgebung des Kerns, als auch in den Fortsätzen; diese gehen ohne sichtbare Grenze in die Zellen über. An feinen Schnitten der Gesehwulst bekommt man den Eindruck, als ob die Zwischensubstanz nur durch die Fortsätze der Zellen gebildet wird. Die Glaskörperm knötchen zeigen ganz dasselbe Aussehen wie die Hauptgeschwalst, sie sind gegen die Umgebung scharf begrenzt; dies sieht man sehr deutlich an den mit Haematoxylin gefärbten Präparaten; einzelne Kuötchen sind ron oinem Hof yon Eiterkörperchen umgeben. Zwischen den Knötchen liegen auf den Glaskörpermembranen äusserst zahlreiche Zellen von dem Aussehen der normalen Glaskörperzellen; Bhatgefässe werden in den Membranen nicht aufgefunden. Die Chorioidea

zu befallen, welche den ersten Herd der Geschwulst beherbergt. Der Primärtumor war schon zu gross, um eine Entscheidung in dieser Hinsicht zu gestatten. In einern früheren Stadium, als in meinem Fall, ist meines Wissens bisher kein Retinagliom zur anatomischen Untersuchngg gelangt. Die Frage nach der zuerst ergriffenen Schicht der Netzhaut bei der Entstehung vou Gliomen darf demnach als eine offene bezeichnet werden. 
und Sclera lassen auch bei der mikroskopischen Untersuchung keine Veränderung erkennen.

Die anatomische Untersuchung des linken Auges hat in dem mitgetheilten Fall ergeben ein Haselnuss grosses, in den Glaskörper prominirendes Gliom im vorderen Abschnitt der inneren Bulbushälfte*), zahlreiche, sehr kleine, disseminirte Herde der äbrigen Retina, die ersten Zeichen von Infiltration der Papille, und unendlich viele, sehr kleine Gliomknoten an feinen Glaskörpermembranen. Auf Grund unserer bis heute gewonnenen Kenntnisse über das Verhalten des Netzhantglioms und mit Rüeksicht auf den Umfang der einzelnen Herde wird man mit grösster Wahrscheinlichkeit annehmen können, dass der Tumor neben der Ora serrata die Primärgeschwulst darstellt, und dass von dieser aus die übrige Retina, die Papille und der Glaskörper inficirt worden sind. Für die disseminirten Infiltrate der Netzhaut und für den kleinen Knoten der Papille wird diese Anschauung kaum auf Widerspruch stossen; sie wird auch für die Erkrankung des Glaskörpers anerkannt werden müssen, wenn man an der, wie es seheint, jetzt allgemein angenommenen Virchow'schen

*) Dex mitgetheilte Fall von Glioma retinae endophytum gleicht auffallend den von Iwanoff (Graefe's Arohiv, XV, 2 , p. 69), Hirschberg (ibid. XVI., 1., p. 296) und Dreschfeld (Mied. Centralbl., 1875, p. 196) beschriebenen. Iwan off war, wie Hirschberg mittheilt, von der Aehnlichkeit seines und des Hirschberg"schen Präparates ïberrascht. Alle vier Falle zeigen einen Geschwulstknoten im vorderen Abschnitt der Retina und zahlreiche disseminirte Herde der übrigen Netzhaut; nur in dem von Hirschberg beobachteten Auge scheint die Bildang eines grösseren Tumors nicht so ausgesprochen gewesen zu sein; in dem von lwanoff und mir untersuchten sass die etwa Haselnuss grosse Geschwulst im nasalen Abschnitt der vorderen Halfte. Dreschfeld hat nicht angegeben, $o b$ die Nenbildung nasal- oder temporalwärts gefunden wurde, 
These festhult, dass das Gliom von dem Stützgewebe der Netzhaut resp. des Centralnervensystems ausgeht. Wir sind dann vor die Frage gestellt, auf welchem Wege die Affection des Glaskörpers entstanden ist, eine Frage, die zugleich diejenige in sich schliesst, warum das Corpus vitreum in der Mehrzahl der Fille von Netzhantgliom nicht inficirt, sondern vielmehr durch die wachsende Geschwulst zu mehr oder weniger vollständigem Schwund gebracht wird. Die Beantwortung dieser Eragen ist von den bisherigen Ergebnissen der pathologisch-anatomischen Untersuchungen uber die Verbreitungswege der heteroplastischen Erkrankung der ubrigen Theile des Augapfels abhängig.

Wenn wir von dem Wachsthum der Geschwulst in der Continuität absehen, so haben wir zuerst die von Knapp aufgestellte Annahme zu erwähnen, dass sich von der Aussenfläche des Glioms einzelne Partikelchen loslösen, auf die Chorioidea fallen und so dieselbe inficiren können. Es erscheint mir sehr zweifelhaft, ob auf dem Wege der sogenannten Keimaussaat eine heteroplastische Affection der Aderhant zu Stande kommt. Die Vorstellung, dass sich von dem Gliom, einer organischen Neubildung, kleine Partikelchen in Folge ron Erweichung einzelner Stellen ohne von aussen einwirkende Kraft loslossen, erseheint mir nicht annehmbar. Abtrennung von Geschwulsttheilchen überhaupt ist ja beobachtet worden; es handelt sich dabei um Tumoren, die in Blutgefässe, namentlich Venen, hineingewachsen sind, und man wird ohne Weiteres zugeben, dass der Blutstrom im Stande ist, kleine Massen von der Oberfläche der Geschwalst abzuspülen*).

*) Als Beispiel von Verbreitung einer Gesehwuist durch Keimaussaat wird von Hirschberg (Der Markschwamm dex Netzhaut 
Ausserdem sollte man erwarten, dass, wenn die Annahme von Knapp richtig wäre, die losgelösten Partikelchen nicht vis-à-vis dem Netzhauttumor auf die Chorioidea gelangen, sondern vermöge ihres höheren specifischen Gewichtes in der subretinalen Flüssigkeit za Boden sinken und, entsprechend den bekannten Präcipitaten auf der hinteren Fläche der Hornhaut, besonders an die unteren Parthien der Chorioidea angeschwemmt werden. Die Knapp'schen Befunde werden meiner Ansicht nach natürlicher in der Weise erklärt, wie Schweigger*) eine von ihm angestellte, dem Knapp'schen entsprechende Beobachtung deutet. Schweigger nimmt für seinen Fall an, dass die kleinen Aderhautherde durch directe Infection von Seiten der drusigen Oberfläche des Netzhauttumors schon zu einer Zeit entstanden sind, in der die Retina noch der Chorioidea anlag, und dass die Retina erst später abgelöst worden ist.

Mag nun die Annahme der heteroplastischen Verbreitung des Netzhautglioms auf dem Wege der Keimaussaat begründet sein oder nicht, die Erkrankung des Corpus vitreum in dem vorliegenden Falle ist schon deshalb auf diesem Wege nicht zu erklären, weil die Oberfläche des Tumors nicht bröckelig aussah.

Man wird von vornherein annehmen dürfen, dass die

Berlin 1869, p. 96) und von Leber (Handb. von Graefe-Saemisch, V., p. 722), das von Virchow (Die krankh. Geschwülste, Berlin 1863, p. 54) angeführte Entstehen multipler Krebseruption im Peritoneum bei Magencarcinom erwähnt. Virchow selbst hält jedoch das Seminium in diesem Falle nicht für absolut sicher (p. 55), und es ist bemerkenswerth, dass weder Cohnheim (Allg. Pathol., Berlin 1877) noch Ziegler (Lehrb. der pathol. Anatomie, 1884 ) in den Kapiteln über die Bildung von Metastasen von Neubildungen die Keimaussaat erwähnen.

*) Graefe's Archiv, VI, 2., p. 328. 
intraoculären Geschwülste auf denselben Bahnen locale oder râumlich mehr getrennte Metastasen*) machen, wie die Neubildungen anderer Korpertheile. Die Metastasen entstehen nach den heute herrschenden Anschauungen aus Geschwulstzellen, die entweder auf der Bahn der Lymphoder Blutgefässe fortgeschwemmt werden, oder vermoge hrer eigenen Locomotionsfuhigkeit fortwandern***).

Für die Netzhautgliome liegt in Bezug auf die Lymphgefässe eine positive Beobachtung von Seiten Dreschfeld's (1.c.) vor, welcher zahlreiche Gliomzellen in den perivaseulären

*) Wenn ich den Ausdruck "Metastasen": auch für solche von dem primären Tumor inducirte Heerde gebrauche, die von demselben räumlich nicht weit getrennt sind, so kann ich mich auf die Autorität Virchow's berufen, der in der 3, Vorlesung seines Werkes über die krankhaften Geschwulste (Berlin 1863) die Worte denteropatisch, secundär und metastatisch ohne strenge Scheidung anwendet, und der ansdrïtcklich sagt: „Die Bildung dieser neuen Herde (sel. in der Umgebung des Tumors), oder, was man kurzweg das Wachsthum der Geschwulst genannt hat, das ist für mich genau dasselbe, wie die Erkrankung der Lymphdrüsen und entfernter Organe im Laufe der Generalisation" (pg. 52). Recklinghausen (Handb. der allg. Pathol. 1883) definirt Metastase, als eine Uebertragung oder Versetzung von pathologischen Stoffen von einem Orte des Körpers auf den anderen." (pg. 164) und legt demnach auf die Grösse der Entfernung des primären und secundären Heerdes kein entscheidendes Gewicht. As seheint mir mehr eine Sache des Uehereinkommens als des Princips zu sein, ob man dem Primärtumor nahe gelegene, von ihm durch ganz normales Gewebe getrennte Herde als secundäre oder metastatische bezeichnet.

**) Virchow hat auf die Möglichkeit, dass Sareomzellen von der Geschwulst in gesunde Theile einwandern kömnen, aufmerksam gemacht (cf. Fuchs, Das Sarcom des Uvealtractns, Wien, 1882, \&. 36, pg. 183 und \&. 39, pg. 196). Auch Cohnheim (Allgemeine Patholologie 1877 pg. 681) hält die Frage für berechtigt, ob bei dem Sarcom die locale Dissemination nicht öfter durch wandernde Sarcomzellen vermittelt wird. 
Lymphscheiden der Retinacapillaren fand. Ich habe eine Anzahl feiner Schnitte von der Grenze des Tumors und solcher mit disseminirten Herden der Retina mit Rücksicht auf das Verhalten der kleinen Gefässe und deren Umgebung untersucht und nichts gefunden, was der Dreschfeld'schen Beschreibung ähnlich gesehen hätte. Dieses negative Ergebniss macht es zum mindesten unwahrscheinlich, dass die Gliomzellen in dem vorliegenden Fall von der Primärgeschwulst auf dem Wege der perivascularen Lymphscheiden zu den kleiven disseminirten Metastasen der Nervenfaserschicht gelangt sind. Wahrscheinlich kann der Transport der Gliomzellen auch auf anderen Bahnen von Statten gehen; ein positiver Befund konnte aber in dieser Hinsicht in meinen Präparaten nicht erhoben werden.

Die in Rede stehenden Fragen können natürlich auf Grund eines einzigen Falles nicht zur Entscheidung gebracht werden. Ich möchte nur noch das hinzufügen, dass, wie mir scheint, die spontane Locomotionsfahigkeit der Geschwulstzellen (die ja überhaupt noch nicht sicher bewiesen ist) bei der heteroplastischen Verbreitung von Neubildungen im Innern des Auges eine sebr untergeordnete resp. gar keine Rolle spielt. Hierfür dürfte die Thatsache sprechen, dass der Regel nach der Glaskörper bei den beiden bekannten Formen der intraocularen Tumoren, den Gliomen und Sarcomen, nicht inficirt wird. Denn, wenn die Gliome oder Sarcome durch spontane Bewegung ihrer Zellen sich verbreiten würden, so sollte man erwarten, dass diese auch häafiger in den Glaskörper gelangen, dass häufiger eine gliomatöse Infiltration des Glaskörpers beobachtet würde. Die Frage, ob das Corpus vitreum eigene, fixe Zellen oder, wie Schwalbe behauptet, nur eingewanderte metamorphosirte weisse Blutkörperchen enthält, dürfte bei dieser Betrachtung nicht ron Belang 
sein, da eine Theilnabme der fixen Organzellen an dem Aufbau der Metastasen heute sowohl im Allgemeinen*) als auch im Speciellen für das Netzhautgliom**) negirt wird.

Aus dem genannten Grunde darf es als unwahrscheinlich bezeichnet werden, dass in dem mitgetheilten Falle die Geschwulstzellen durch eigene Locomotionsfähigkeit in den Glaskörper gelangt sind.

Gegen diese Deduction könnte der Einwand erhoben werden, dass zwar Gliom- oder Sarcomzellen in den Glaskörper einwandern, aber wegen des Mangels desselben an Blutgefässen sich nicht zu Metastasen entwickeln. Der Einwurf scheint mir durch das vollkommene Fehlen von Blutgefässen in den Glaskörperherden des mitgetheilten Falles widerlegt zu werden. Denn dieses sehr bemerkenswerthe Verhalten zeigt, dass eine heteroplastische Affection des Corpus vitreum zu Stande kommen kann, ohne dass Blutgefässe in die einzelnen Herde eintreten ***) Dabei ist aber nicht ausgeschlossen, dass Blutgefasse bei der ersten Entstehung der fraglichen Erkrankung eine Rolle gespielt haben. Im Gegentheil scheint mir die Entstehungsart der bei meinem Patienten beobachteten Glaskörpermetastase dem Verständniss näher gerückt zu

*) Cohnheim, Allg. Pathologie 1877, 1, p.670, und Ziegler Lehrb. der pathol. Anat., 1884, I., p. 197.

**) Nach Knapp tritt bei der metastatischen Erkrankung der Chorioidea keine Wucherung der pigmentirten Stromazellen auf; diese Thatsache ist durch Leber (Handb. von GraefeSaemisch, V., p. 722) bestätigt worden.

***) Es darf darauf hingewiesen werden, dass auch bei der ophthalmoskopischen Untersuchung, ebensowenig wie bei der mikroskopischen Blutgefässe bemerkt wurden. 
werden durch die Annahme, dass das Gliom sich schon im intrauterinen Leben entwickelt hat und zwar zu einer Zeit, wo der Glaskörper noch vaseularisirt war, und dass die fötalen Glaskörpergefässe die Bahn darstellen, auf welcher die Gliomzellen in das Corpus vitreum gelangt sind. Nach den Angaben der Mntter ist es wahrscheinlich, dass das Gliom auf dem linken Auge congenital war; dasselbe darf deshalb für das rechte Auge angenommen werden, da congenitale Gliome fast immer doppelseitig sind*). Die Geschwulst müsste auf dem rechten Auge bei der Geburt kleiner als auf dem linken gewesen und sehr langsam gewachsen sein. $\mathrm{Zu}$ Gunsten der fraglichen Entstehungsart der Glaskörperaffection kann auf die Beobachtung hingewiesen werden, das die mit Gesehwulstknötchen besetzten Glaskörpermembranen besonders in der Nähe der hinteren Linsenand der vorderen Netzhautfläche gefunden wurden, also in den Theilen des Augapfels, wo sich die fötalen Glaskörpergefässe verbreiten. Die letzteren müssten sich dann ebenso zurückgebildet haben, wie es sonst der Fall ist; hierin könnte man vielleicht den Grund sehen, warum die Knötchen keinen grösseren Umfang erreicht haben; denn Blutgefässe stellen einen unumgänglich nothwendigen Bestand theil bei der Entwickelung umfangreicherer Tumoren dar.

Die in Rede stehende Erklärung würde es verständlich machen, warum bei intraocularen Geschwülsten, die sich im extrauterinen Leben entwickeln, secundäre Erkrankung des Glaskörpers nicht beobachtet wird.

Alle anderen oben genannten Wege der heteroplasti-

*) Lueber, Handb. von Graefe-Sämisch V. pag. 732. 
schen Verbreitung stehell dem Sarcomen ebenso zur Verfügung wie den Gliomen; bei den Gliomen allein, von denen jedenfalls ein grosser Theil congenital ist, ist die Möglichkeit gegeben, dass Geschwulstzellen auf der Bahn der fötalen Glaskörpergefässe in das Corpus vitreum gelangen können. 